\title{
A PRECISE MEASUREMENT OF THE REAL PART OF THE ELASTIC SCATTERING AMPLITUDE
}

\section{AT THE S̄̄ps}

\author{
UA4/2 Collaboration \\ Genova $^{a}$ - Palaiseau $^{b}-$ Praha $^{c}-$ Roma $^{d}-$ Valencia $^{e}$
}

C. Augier ${ }^{b}$, D. Bernard ${ }^{b}$, J. Bourotte ${ }^{b}$, M. Bozzo $^{a}$, A. Bueno ${ }^{e}$,

R. Cases $^{e}$, F. Djama ${ }^{e}$, M. Haguenauer ${ }^{b}$, V. Kundrát ${ }^{c}$, M. Lokajíček ${ }^{c}$,

G. Matthiae ${ }^{d}$, A. Morelli ${ }^{a}$, F. Natali ${ }^{d}$, S. Němeček ${ }^{c}$, M. Novák ${ }^{c}$,

E. Sanchis ${ }^{e}$, G. Sette ${ }^{a}$, M. Smižanskác, J. Velasco ${ }^{e}$

${ }^{a}$ Università di Genova and Sezione INFN, Genoa, Italy

${ }^{b}$ Ecole Polytechnique/IN2P3-CNRS, Palaiseau, France ${ }^{c} A V \check{C} R$, Institute of Physics, Praha, Czech Republic

${ }^{d}$ Università di Roma II and Sezione INFN, Roma, Italy

${ }^{e}$ IFIC-Centro Mixto Universidad de Valencia-CSIC, Valencia, Spain

\begin{abstract}
:
A precise measurement of $\bar{p} p$ elastic scattering in the Coulomb-strong interaction interference region was performed at the CERN S̄ppS Collider at a centre-of-mass energy of $541 \mathrm{GeV}$. The ratio of the real to the imaginary part of the forward elastic scattering amplitude was found $\rho=0.135 \pm 0.015$. The slope of the exponential fall off of the strong interaction part was also measured, $b=15.5 \pm 0.1 \mathrm{GeV}^{-2}$.
\end{abstract}

\section{GENEVA}

1993 

The surprisingly high value of the ratio $\rho$ of the real to the imaginary part of the forward elastic scattering amplitude obtained by the UA4 Collaboration [1] had given rise to various theoretical interpretations. It is worth recalling that the UA4 result was obtained in a single S $\bar{p} p S$ run of about two days and systematic effects could not be thoroughly studied. The remarkable improvements in luminosity and beam optics of the S̄ppS Collider since 1986, opened the possibility for a new measurement with a substantial reduction of the statistical uncertainty and of the sources of possible systematic errors.

At high energy the scattering amplitude is mainly imaginary. A knowledge of the real part allows to get predictions of the total cross section at still higher energies by using dispersion relations. The real part of the strong scattering amplitude is obtained by measuring the differential cross section in the region where strong and Coulomb interactions interfere. The standard method is described in ref. [1] and here only the basic features are recalled.

The differential cross section for $\bar{p} p$ scattering can be written as:

$$
\begin{aligned}
& \frac{d \sigma}{d t}=\frac{1}{\pi}\left|f_{C}+f_{S}\right|^{2} \\
& \text { where } \quad f_{C}=\frac{2 \pi \hbar c \alpha G^{2}(t) e^{-i \alpha \phi(t)}}{|t|} \\
& \text { and } \quad f_{S}=\frac{(\rho+i) \sigma_{t o t} e^{-b|t| / 2}}{4 \pi \hbar c}
\end{aligned}
$$

$\mathrm{G}(\mathrm{t})$ is the proton electromagnetic form factor, $\alpha$ is the fine structure constant and $\phi$ is the commonly used Coulomb phase [2]. The relative maximum of interference occurs at four momentum transfer squared $|t|=8 \pi \alpha(\hbar c)^{2} / \sigma_{\text {tot }} \approx$ $1.1 \times 10^{-3} \mathrm{GeV}^{2}$, corresponding at $\sqrt{s}=541 \mathrm{GeV}$ to a scattering angle of $\approx 120$ $\mu \mathrm{rad}$.

To reduce possible systematic error sources, it is important to measure elastic scattering below the relative interference maximum. Since the minimum $|t|$ at which the measurement is feasible is proportional to $1 / \beta^{*}$, where $\beta^{*}$ is the betatron function at the $\bar{p} p$ crossing point, a special super-high $\beta^{*}$ insertion was designed [3] for the SpppS Collider. A value for $\beta_{H}^{*}$ as high as $2500 \mathrm{~m}$ was obtained in the horizontal plane, together with $\beta_{V}^{*}=87 \mathrm{~m}$, by doubling the standard machine quadrupoles QF16 and QF20. In order to obtain a symmetric optics and at the same time to position the crossing point in a region without magnetic field, the quadrupole QF18 was also doubled, leaving a $3 \mathrm{~m}$ long free field space in the middle of the straight section, where the crossing point was located. 


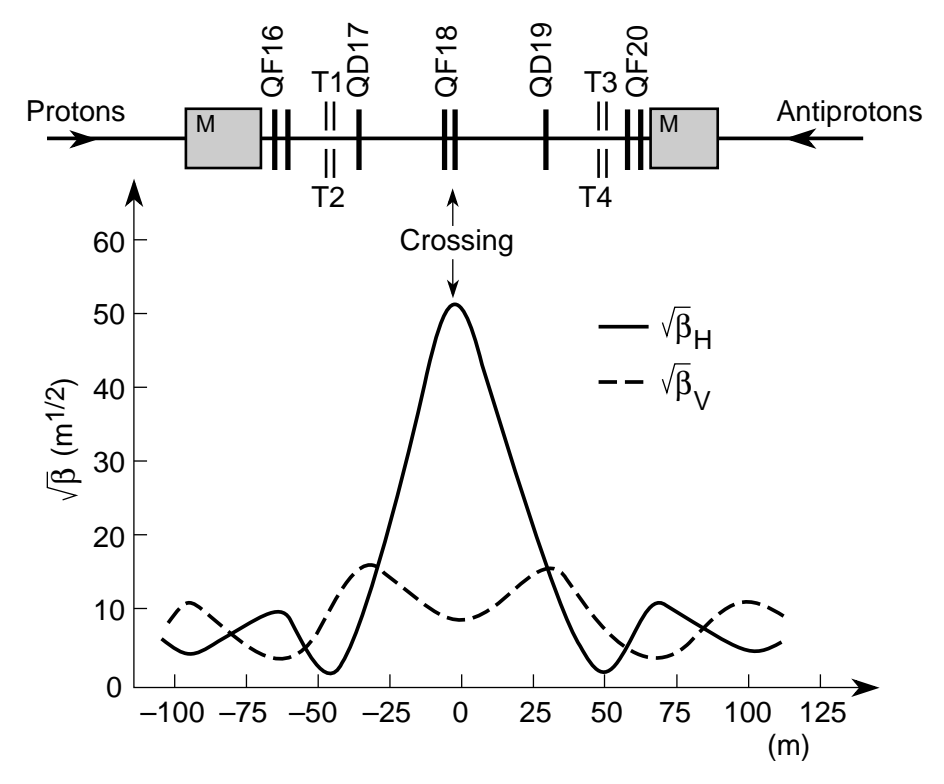

Figure 1:

Beam optics and experimental layout

The position of the crossing point was monitored by recording the time of flight difference between planes of counters placed symmetrically on both sides of the crossing point.

The scattered antiprotons (respectively protons) traverse QD17 (QD19) before reaching the detectors placed at the focal point corresponding to a horizontal betatron phase advance of $\pi / 2$. This allows a unique correspondence in the horizontal plane between the scattering angle and the displacement of the trajectory at the detectors, irrespective of the size and position of the crossing. The "effective distances" are $L_{H}^{\bar{p}} \approx L_{H}^{p} \approx 65 \mathrm{~m}$, and $L_{V}^{\bar{p}} \approx L_{V}^{p} \approx 27 \mathrm{~m}$.

The scattered proton and antiproton were detected by a system of four telescopes, T1 to T4, placed symmetrically on both sides of the intersection region as shown in fig.1. Each telescope was composed of two "Roman pots", $0.73 \mathrm{~m}$ apart, placed symmetrically around the point where the horizontal betatron phase advance is $\pi / 2$. An elastic event was defined as one of the combinations $\mathrm{C} 1=$ $\mathrm{T} 1 * \mathrm{~T} 4$ or $\mathrm{C} 2=\mathrm{T} 2 * \mathrm{~T} 3$.

The detectors for this experiment are conceptually similar to those already used for the measurement of the total cross section and of $\rho$ by the former UA4 collaboration[4]. However, various modifications were implemented in order to reduce systematic uncertainties as, a new system for the survey, new detectors for the measurement of the vertical coordinate and an improvement of the me- 
chanics to reduce dead space at the edge of the detectors close to the beam.

Each "Roman pot" was equipped with a four planes drift chamber to measure the horizontal coordinate of the scattered particle, a set of twelve staggered scintillating fiber planes for the measurement of the vertical coordinate, a hodoscope with eight "finger" counters and a trigger counter seen by two independent photomultipliers. Three mechanical gauges were provided for each pair of facing pots in order to measure with remote control their distance with an accuracy of 10 $\mu \mathrm{m}$. A full survey of all detectors, including the position of each single drift sense wire, has been done. The overall remaining scale uncertainty was reduced to $\approx$ $20 \mu \mathrm{m}$. Detailed information about the "Roman pots" and the detectors can be found in ref.[5].

The present measurement was performed in a five weeks dedicated S̄ppS run. By setting the machine collimators to a distance of about four r.m.s. of the horizontal beam size, clean beams were obtained. The "Roman pots" were set such that the background was still tolerable in the drift chambers. The closest sensitive edge of the detectors could be brought, during the period of stable beams, to $4 \mathrm{~mm}$ from the beam axis, which corresponded to a minimum momentum transfer $|t|=3 \times 10^{-4} \mathrm{GeV}^{2}$. A total of $13 \mathrm{~S} \bar{p} p S$ independent runs, with an average luminosity of $3 \times 10^{26} \mathrm{~cm}^{-2} \mathrm{~s}^{-1}$ were used for data taking. With a trigger rate of $\approx 3 \mathrm{~Hz}$, about $1.5 \times 10^{6}$ triggers were collected of which an average of $65 \%$ were elastic events.

A wire scan system [6] placed at the crossing point allowed a precise measurement of both horizontal and vertical beam profiles and positions. Typical r.m.s. values were found to be $5 \mathrm{~mm}$ and $1 \mathrm{~mm}$ in the horizontal and vertical planes respectively. The corresponding angular spreads are $4 \mu \mathrm{rad}$ and $15 \mu \mathrm{rad}$ respectively. Care was put to have both $\bar{p}$ and $p$ bunches of similar size and content in order to ensure maximum beam stability.

The data analysis was performed in the following steps:

1. The calibration of the drift chambers was done using the elastic scattering events themselves. The resulting resolution of $\approx 150 \mu \mathrm{m}$ per drift plane, was found to be constant over the detector range. The detection efficiency was determined as a function of the horizontal (x) coordinate. A reduction of efficiency was observed near the sense wires and the cell separations. Track reconstruction was performed by demanding at least 4 out of the 8 drift planes in each telescope. In any case at least one plane per chamber had to show a hit. With this selection the average telescope efficiency was measured as a function of $\mathrm{x}$-coordinate and was found $0.995 \pm 0.002$.

2. The vertical (y) resolution was obtained by comparing the distributions of the reconstructed coordinate in the two detectors of the same telescope. The measured resolution of $60 \mu \mathrm{m}$ is definitely better than the basic needs of the 


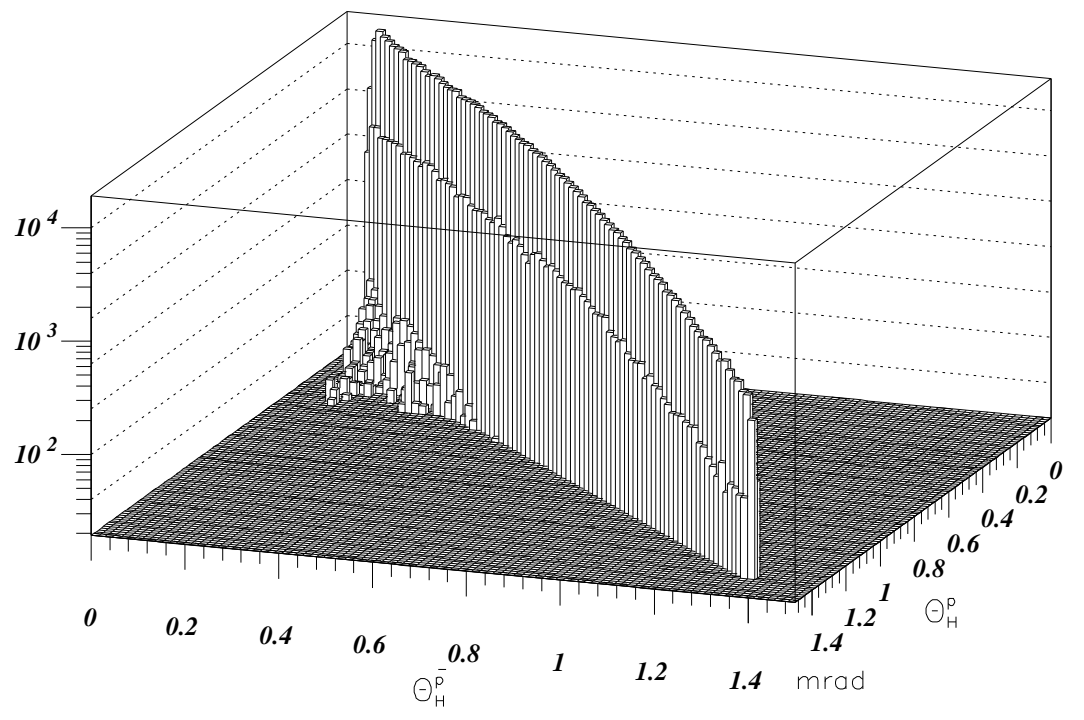

Figure 2:

Collinearity distribution in the horizontal plane

experiment. No sizeable inefficiency was detected in the inner pots. The secondary interactions of the scattered protons or antiprotons were responsible for a $1.5 \%$ inefficiency in the outer pots, due to the limitation of the multitrack reconstruction. The non-uniformity for all detectors was $<0.2 \%$.

3. The trigger counters behaviour was checked by measuring the pulse height in each of the two photomultipliers. A study as a function of the coordinate showed that uniformity over the entire area was better than $99.8 \%$.

4. After track reconstruction, the following selection criteria have been applied:

- Cut on the collinearity distribution in the horizontal plane, $\left|x_{\bar{p}} / L_{H}^{\bar{p}}-x_{p} / L_{H}^{p}\right|<14 \mu \mathrm{rad}$. The collinearity distribution is shown in fig.2. The r.m.s. of $\approx 5 \mu \mathrm{rad}$ reflects mainly the horizontal angular dispersion of the beam.

- Cut on the collinearity distribution in the vertical plane, $\left|y_{\bar{p}} / L_{V}^{\bar{p}}-y_{p} / L_{V}^{p}\right|<214 \mu \mathrm{rad}$. The much wider cut applied in the vertical plane reflects the dependence of the $y$-coordinate at the detectors on the vertical size of the beam at the crossing point.

5. To select a safe region of acceptance for both $\bar{p}$ and $p$ telescopes, fiducial cuts of $1 \mathrm{~mm}$ at the edge near the beam and $\approx 2 \mathrm{~mm}$ away from the 


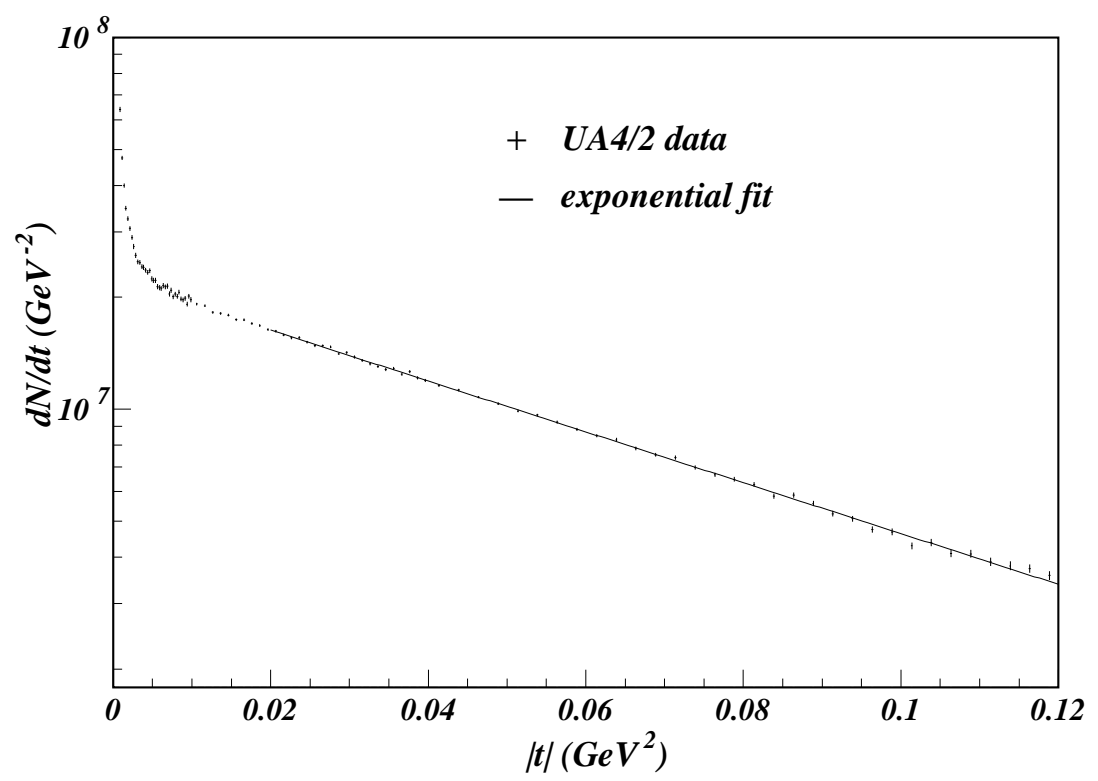

Figure 3:

Differential $d N / d|t|$ distribution in the full $|t|$ range The line corresponds to a pure exponential fit

shadow of the quadrupole vacuum chamber were applied at the detector level. The t-range was $1 . \times 10^{-3}<|t|<0.12 \mathrm{GeV}^{2}$ for the combination $\mathrm{C} 1$ and $0.75 \times 10^{-3}<|t|<0.12 \mathrm{GeV}^{2}$ for the combination $\mathrm{C} 2$. The total number of elastic events was $7.85 \times 10^{5}$.

6. A Monte-Carlo simulation has been performed to compute the detector acceptance taking into account all machine parameters (beam position, size, angular spread, optics) and the detector parameters (position, resolution, efficiency). The resulting acceptance is very close to the pure geometrical one. The overall momentum transfer resolution $\Delta t \approx 0.0025 \sqrt{|t|}$ is in agreement with the observed width of the collinearity distributions.

7. The background was determined by studying the $\left(\theta_{H}^{\bar{p}}, \theta_{H}^{p}\right)$ correlation. It has been found that the background is constant for fixed $\left(\theta_{H}^{\bar{p}}+\theta_{H}^{p}\right)$. The background level increases from $0.02 \%$ at large $|t|$, to $1.5 \%$ in the first $|t|$-interval considered. At large $|t|$ it is compatible with the result of a Monte-Carlo calculation based on diffractive events. At small $|t|$ it is mainly induced by random events from the beam halo.

Despite the very good setting of the beam orbit, a small residual angle of 
the beam with respect to the nominal machine axis was still present. The vertical beam angle $\alpha_{V}$ was evaluated by demanding up/down symmetry for the y-distribution of the events. It was found $\alpha_{V}=7 \pm 5 \mu \mathrm{rad}$, which has a negligible effect on the final $|t|$ distribution. The residual horizontal crossing angle was obtained by imposing in the fitting procedure the same values of the parameters $b$ and $\rho$ for both combinations $\mathrm{C} 1$ and $\mathrm{C} 2$. The angle $\alpha_{H}$ was found $\alpha_{H}=-1 \pm$ $1 \mu \mathrm{rad}$. This uncertainty is taken into account in the evaluation of the final error.

The final $|t|$-distribution of elastic scattering $\mathrm{dN} / \mathrm{d}|t|$, corrected for the experimental acceptance is shown in fig.3. Clear evidence for the Coulomb rise at low $|t|$ is observed. The experimental results are also presented in table 2, where $\mathrm{dN} / \mathrm{d}|t|$ is given as a function of $|t|$.

The data were fitted using the theoretical form given by eqs 1 to 3 , both by $\chi^{2}$ minimization and maximum likelihood method, keeping the absolute normalization free. By leaving all the parameters free, the fitted value of the total cross section is $\sigma_{t o t}=(63 \pm 1.5$ (stat.) \pm 3 (syst.) $\mathrm{mb}$. The sytematic error is mainly due to the sensitivity of the pure Coulomb part to the uncertainty in the acceptance at low $|t|$.

In order to extract the real part of the amplitude, the more precise result of a previous measurement by UA4 [7], $\left(1+\rho^{2}\right) \sigma_{\text {tot }}=63.3 \pm 1.5 \mathrm{mb}$ was used. The fit in the full $|t|$-range $\left(0.75 \times 10^{-3}<|t|<0.12 \mathrm{GeV}^{2}\right)$ gives $\rho=0.135 \pm 0.007$ and $b=15.52 \pm 0.07 \mathrm{GeV}^{-2}$, the quoted errors being statistical. The value of the $\chi^{2} /$ d.o.f. was equal to 1.1 for the entire $|t|$ range, and to 1.3 if the evaluation is restricted to the interference region $\left(|t| \leq 0.01 \mathrm{GeV}^{2}\right)$. The $d N / d|t|$ distribution in the interference region is shown in fig.4.

A fit in the interval $0.05<|t|<0.12 \mathrm{GeV}^{2}$, where the correlation between the parameters $\rho$ and $b$ is practically absent, gave the result $b=15.5 \pm 0.2$ $\mathrm{GeV}^{-2}$. An attempt was also made to detect a possible deviation from a single exponential fall off of the strong interaction part of the differential cross section by including a quadratic term, $e^{-\left(b|t|+c t^{2}\right)}$, in the exponential dependence of the strong amplitude. The result of this last fit gave $c=1 \pm 1 \mathrm{GeV}^{-4}$, which doesn't favour such an hypothesis.

The correlation between $b$ and $\rho$ was derived from the fitting procedure and is given by $\delta \rho / \delta b=-0.072 \mathrm{GeV}^{2}$. The correlation between $\sigma_{\text {tot }}$ and $\rho$ is $\delta \rho / \delta \sigma_{\text {tot }}$ $=0.0082 \mathrm{mb}^{-1}$. The error on $\rho$ due to the angular scale uncertainty is $\Delta \rho=0.003$.

It should be mentioned that the $|t|$-scale error due to the uncertainty on the beam momentum can be neglected. In fact a very accurate determination of the beam momentum, $270.5 \pm 0.1 \mathrm{GeV} / \mathrm{c}$, became available recently by measuring 


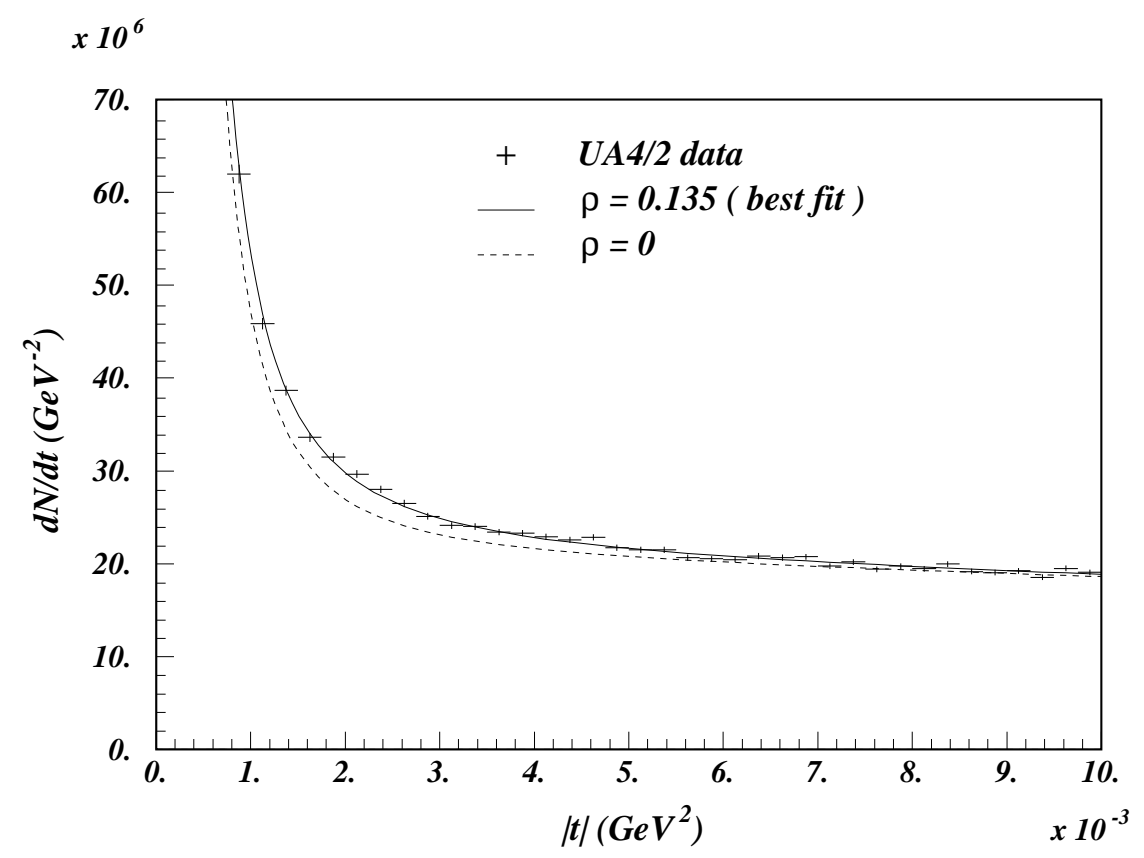

Figure 4:

Differential $d N / d|t|$ distribution in the interference region The upper line corresponds to the best fit with $\rho=0.135$, the lower line to the case $\rho=0$

the difference of revolution frequency in the SPS between protons and ${ }^{16} \mathrm{O}$ ions[8].

The uncertainty coming from the acceptance calculation estimated by making several $\mathrm{x}_{\min }$ cuts was found to be $\Delta \rho=0.01$. By assuming a $50 \%$ uncertainty on the background determination, the measurement of $\rho$ would change by 0.005 . The error derived from beam position and angle is $\Delta \rho=0.005$. The various sources of uncertainties are summarized in table 1.

\begin{tabular}{||c|c||}
\hline Error source & $\Delta \rho$ \\
\hline Acceptance & 0.010 \\
\hline Statistics & 0.007 \\
\hline Beam position/angle & 0.005 \\
\hline Background & 0.005 \\
\hline Angular scale & 0.003 \\
\hline Beam momentum & - \\
\hline
\end{tabular}

Table 1 
A quadratic combination of all the uncertainties mentioned above leads to the final value of:

$$
\rho=0.135 \pm 0.015
$$

The present result was obtained assuming an equal slope $b$ for the real and imaginary parts of the strong amplitude. Nevertheless, if the exponential fall off of the real part would be twice as fast as the imaginary part, $\rho$ would be lowered by 0.02 . The effect induced on $\rho$ for using different expressions from the literature of the electromagnetic proton form factor and of the Coulomb phase [9] was less than 0.005 .

This result is definitely in the range of the standard predictions [10]. At present energies, it implies that there is no sizeable contribution of the odd under crossing part of the forward amplitude which was discussed on general grouds and, recently, in the framework of perturbative-QCD [11]. The previous result $(\rho=0.24 \pm 0.04$ ) obtained with a poor beam optics, a factor eleven less statistics and much less control of systematic effects should be considered as superseded.

We wish to thank very warmly A.Martin for his continuous support. The design of the beam parameters as well as clean operation conditions were an essential part of this experiment. This experiment couldn't have been successful without the full participation and hard work of all the persons involved in the accelerator complex: ACOL, PS, SPS. We would like to express special thanks to P.Faugeras, A.Faugier, A.Hilaire, J.B.Jeanneret, A.Lacourt and T.Linnecar. The collaboration of the technicians at AVČR, IFIC, INFN, IN2P3, is warmly acknowledged. We wish also to thank R.Maleyran for the design of the "Roman pots". 


\begin{tabular}{|c|c|c|c|c|c|}
\hline $\begin{array}{l}|t| \text {-interval } \\
10^{-3} \mathrm{GeV}^{2}\end{array}$ & $\begin{array}{c}d N / d|t| \\
10^{4} \text { events } / \mathrm{GeV}^{2}\end{array}$ & $\begin{array}{c}\Delta(d N / d|t|) \\
10^{4} \text { events/GeV } / \mathrm{GeV}^{2}\end{array}$ & $\begin{array}{l}|t| \text {-interval } \\
10^{-3} \mathrm{GeV}^{2}\end{array}$ & $\begin{array}{c}d N / d|t| \\
10^{4} \text { events/GeV } / \mathrm{GeV}^{2}\end{array}$ & $\begin{array}{c}\Delta(d N / d|t|) \\
10^{4} \text { events } / \mathrm{GeV}^{2}\end{array}$ \\
\hline $0.75-1.00$ & 6402. & 107. & $23.0-24.0$ & 1558. & 13. \\
\hline $1.00-1.25$ & 4739. & 63. & $24.0-25.0$ & 1516. & 13. \\
\hline $1.25-1.50$ & 3994. & 54. & $25.0-26.0$ & 1482. & 13. \\
\hline $1.50-1.75$ & 3471. & 49. & $26.0-27.0$ & 1482. & 13. \\
\hline $1.75-2.00$ & 3253 . & 46. & $27.0-28.0$ & 1470. & 13. \\
\hline $2.00-2.25$ & 3065 . & 44. & $28.0-29.0$ & 1411. & 12. \\
\hline $2.25-2.50$ & 2895. & 42. & $29.0-30.0$ & 1420. & 13. \\
\hline $2.50-2.75$ & 2739 . & 40. & $30.0-31.0$ & 1382. & 12. \\
\hline $2.75-3.00$ & 2594 . & 39. & $31.0-32.0$ & 1353. & 12. \\
\hline $3.00-3.25$ & 2493. & 37. & $32.0-33.0$ & 1323. & 12. \\
\hline $3.25-3.50$ & 2480 . & 37. & $33.0-34.0$ & 1302. & 12. \\
\hline $3.50-3.75$ & 2418. & 36. & $34.0-35.0$ & 1281. & 12. \\
\hline $3.75-4.00$ & 2408. & 36. & $35.0-36.0$ & 1289. & 13. \\
\hline $4.00-4.25$ & 2367 . & 36. & $36.0-37.0$ & 1244. & 13. \\
\hline $4.25-4.50$ & 2333. & 35. & $37.0-38.0$ & 1263. & 13. \\
\hline $4.50-4.75$ & 2358. & 35. & $38.0-39.0$ & 1215. & 13. \\
\hline $4.75-5.00$ & 2244 . & 34. & $39.0-40.0$ & 1195. & 13. \\
\hline $5.00-5.25$ & 2220 . & 34. & $40.0-42.5$ & 1156. & 8. \\
\hline $5.25-5.50$ & 2221. & 34. & $42.5-45.0$ & 1126. & 8. \\
\hline $5.50-5.75$ & 2135. & 33. & $45.0-47.5$ & 1078. & 8. \\
\hline $5.75-6.00$ & 2122. & 33. & $47.5-50.0$ & 1034. & 8. \\
\hline $6.00-6.25$ & 2114. & 33. & $50.0-52.5$ & 989. & 8. \\
\hline $6.25-6.50$ & 2151. & 33. & $52.5-55.0$ & 964. & 8. \\
\hline $6.50-6.75$ & 2135. & 33. & $55.0-57.5$ & 923. & 8. \\
\hline $6.75-7.00$ & 2145. & 33. & $57.5-60.0$ & 882. & 8. \\
\hline $7.00-7.25$ & 2041. & 32. & $60.0-62.5$ & 849. & 8. \\
\hline $7.25-7.50$ & 2088. & 32. & $62.5-65.0$ & 828. & 9. \\
\hline $7.50-7.75$ & 2006. & 31. & $65.0-67.5$ & 785. & 9. \\
\hline $7.75-8.00$ & 2039 . & 32. & $67.5-70.0$ & 754. & 9. \\
\hline $8.00-8.25$ & 2012. & 31. & $70.0-72.5$ & 742. & 9. \\
\hline $8.25-8.50$ & 2065. & 32. & $72.5-75.0$ & 696. & 9. \\
\hline $8.50-8.75$ & 1980. & 31. & $75.0-77.5$ & 666. & 9. \\
\hline $8.75-9.00$ & 1969. & 31. & $77.5-80.0$ & 648. & 9. \\
\hline $9.00-9.25$ & 1988. & 31. & $80.0-82.5$ & 627. & 9. \\
\hline $9.25-9.50$ & 1917. & 30. & $82.5-85.0$ & 584. & 9. \\
\hline $9.50-9.75$ & 2014. & 31. & $85.0-87.5$ & 587. & 10. \\
\hline $9.75-10.0$ & 1970. & 31. & $87.5-90.0$ & 558. & 10. \\
\hline $10.0-11.0$ & 1919. & 15. & $90.0-92.5$ & 524. & 9. \\
\hline $11.0-12.0$ & 1898. & 15. & $92.5-95.0$ & 507. & 10. \\
\hline $12.0-13.0$ & 1822. & 15. & $95.0-97.5$ & 475. & 9. \\
\hline $13.0-14.0$ & 1811. & 14. & $97.5-100.0$ & 468. & 10. \\
\hline $14.0-15.0$ & 1791. & 14. & $100.0-102.5$ & 429. & 9. \\
\hline $15.0-16.0$ & 1741. & 14. & $102.5-105.0$ & 438. & 10. \\
\hline $16.0-17.0$ & 1739. & 14. & $105.0-107.5$ & 409. & 10. \\
\hline $17.0-18.0$ & 1700. & 14. & $107.5-110.0$ & 408. & 10. \\
\hline $18.0-19.0$ & 1678. & 14. & $110.0-112.5$ & 389. & 10. \\
\hline $19.0-20.0$ & 1638. & 14. & $112.5-115.0$ & 379. & 10. \\
\hline $20.0-21.0$ & 1623. & 14. & $115.0-117.5$ & 373. & 10. \\
\hline $21.0-22.0$ & 1584. & 13. & $117.5-120.0$ & 357. & 10. \\
\hline $22.0-23.0$ & 1555. & 13. & & & \\
\hline
\end{tabular}

Table 2 


\section{References}

[1] UA4 Collaboration, D.Bernard et al., Phys. Lett. B198 (1987) 583

[2] G.B.West and D.R.Yennie, Phys. Rev. D172 (1968) 1413

N.H.Buttimore, E.Gotsman and E.Leader, Phys. Rev. D18 (1978) 694 and Phys. Rev. D35 (1987) 407

R.Cahn, Zeitschr. für Phys. C15 (1982) 253

[3] P.E.Faugeras, "New insertion optics for the Sp̄pS Collider", CERN MT DI/90-5 J.B.Jeanneret, CERN/SPS Internal report

[4] UA4 Collaboration, R.Battiston et al., Nucl. Instrum. Methods A238 (1985) 35

[5] J.Buskens et al., Nucl. Instrum. Methods A207 (1983) 365

UA4/2 Collaboration, C.Augier et al., to be submitted to Nucl. Instrum. Methods A

[6] J.Bosser et al., Nucl. Instrum. Methods A235 (1985) 689

[7] UA4 Collaboration, M.Bozzo et al., Phys. Lett. B147 (1984) 392

[8] X.Altuna et al., CERN SL/92-32 1992

[9] F.Borkowski et al., Nucl. Phys. B93 (1975) 385 and ref. 2 above.

[10] C.Bourrely and A.Martin, Proceedings of the LHC workshop, Lausanne (1984) 323

A.Donnachie and P.V.Landshoff, Nucl. Phys. B244 (1984) 322

R.J.Glauber and J.Velasco, Phys. Lett. B147 (1984) 380

R.Henzi and P.Valin, Phys. Lett. B149 (1984) 239

C.Bourrely, J.Soffer, T.T.Wu, Phys. Lett. B196 (1987) 237

P.Gauron, E.Leader, B.Nicolescu, Nucl. Phys. B299 (1988) 640

M.Block and R.Cahn, Phys. Lett. B188 (1987) 143

S.Hadjitheodoridis and K.Kang, Phys. Lett. B208 (1988) 135

P.Kroll and W.Schweiger, Nucl. Phys. A503 (1989) 865

P.Desgrolard, M.Giffon, L.Jenkovszky, Zeit. Phys. C55 (1992) 637

R.J.M.Covolan et al., Zeit. Phys. C58 (1993) 109

[11] L.Lukaszuk and B.Nicolescu, Nuov. Cim. Lett. 8 (1973) 405

P.Gauron, L.Lipatov and B.Nicolescu, Phys. Lett. B304 (1993) 334 